\title{
IN-LIQUID PLASMA USING MICROWAVE POWER FOR APPLICATIONS
}

\author{
S. Sawada and S. Horikoshi
}

Sophia University, 7-1 Kioi-cho, Chiyoda-ku, Tokyo

sawada-1112@eagle.sophia.ac.jp,horikosi@sophia.ac.jp

Keywords: in-liquid plasma, semiconductor microwave generator, pulse irradiation, wastewater treatment, crosslinking agent/initiator-free polymer-gel synthesis

\begin{abstract}
The research of in-liquid plasma that uses microwave as an energy source can be generated without depending on the electric conductivity of the solution, so it has wide application. However, it is not practical because the microwave antenna (plasma electrode) is deteriorated immediately. We were noticed that the microwave is pulsed to solve this problem. In this study, its optimum conditions was investigated. The best parameters of pulse microwave power was investigated using the generator (peak power; $380 \mathrm{~W}$, pulse width ; $13.4 \mathrm{msec}$ and pulse duty cycle ; $16.7 \mathrm{msec}$ ). Stable in-liquid plasma could be generated in this conditions. This optimised system of in-liquid plasma tried to use applications for wastewater treatment and gel synthesis. In first application (wastewater treatment), tetrabromobisphenol A (TBBPA) was ionized and minerated by using in-liquid plasma method for only $20 \mathrm{~min}$, respectivily. In second one (gel synthesis), in-liquid plasma can synthesize gel-synthesis without crosslinking agent and initiator. This new method could synthesize gel of dimethyl polysiloxane (silicone) and polyvinilypirroridone (PVP) without both crosslinking agent and initiator, it suggested that in-liquid plasma method is effective as a green chemistry process.
\end{abstract}

\section{Introduction}

Historically, in-liquid plasma was generated in electrolytic aqueous media in the late 1980s by Clements et al. [1] who used needle and plate electrodes together with an alternating current (AC) power supply under gas bubbling conditions and was then proposed as a means of water treatments. Studies followed on generating in-liquid plasma by a discharge device using direct current (DC) or an AC power supply and two electrodes [2]. However, many of these devices required the conductive solution medium to generate inliquid plasma. Over a decade ago, Nomura et al. [3, 4] observed the ignition and maintenance of stable plasma in a liquid hydrocarbon solvent exposed to a combination of ultrasonic waves and microwave radiation. Though one of the problems in generating plasma continuously in a liquid medium with microwave radiation at high-applied microwave power levels is the deterioration of the tungsten antenna tip because both plasma heating, which can reach a temperature above the melting point of tungsten $\left(3422{ }^{\circ} \mathrm{C}\right)$, together with ultrasound waves, melted the electrode. In the last few years, our interest in this area has focused on finding ways (our challenges) to improve on the generation of stable in-liquid plasma in aqueous media and its applications. In this regard, the first challenge resolved successfully [5] was reduction of the electrical power from 700 to $220 \mathrm{~W}$ to produce the microwave radiation load on the electrode through the utilization of an integrated hybrid antenna system consisting of a supersonic horn $(19.5 \mathrm{kHz})$ and a microwave radiation $(2.45$ $\mathrm{GHz}$ ) source [5]. Unfortunately, while it was possible to continuous generate the in-liquid plasma; it was not possible to bring about improvements on the complex device. The second challenge resolved was that, in the absence of the supersonic horn and by reducing the 
pressure inside the reaction vessel with an aspirator, the irradiation power of the microwaves could be low significantly from 700 to $150 \mathrm{~W}$ [6]. Most importantly, the latter method led to the generation of stable, permanent in-liquid plasma. In order to advance the miniaturization of the device, we replaced the magnetron microwave generator used earlier [6] by a semiconductor microwave generator and discovered that, unlike the former, irradiation with 150-W microwaves no longer generated in-liquid plasma. Even if the microwave power increased to $300 \mathrm{~W}$, no in-liquid plasma generated-a curious result that led us to query the causes that might explain these observations.

To the extent that a semiconductor microwave generator, by its very nature, emits continuous microwaves that pose a problem in generating in-liquid plasma, we herein reexamine this issue by using a prototype high power semiconductor generator and compare its characteristics to the pulsed microwaves emitted by a magnetron to generate in-liquid plasma [7]. Accordingly, the aim of the present study was to investigate the optimal pulsed microwave conditions to generate long-lived and stable in-liquid plasma, and its applications toward the degradation of a TBBPA wastewater [8], and gel-synthesis of PVP and silicone [9].

\section{Experimental methods}

\subsection{Development of in-liquid plasma device}

Photograph and schematic illustration of in-liquid plasma device are shown in Fig. 1a and 1b, while Fig. 1c displays the photograph of the microwave-generated in-liquid plasma [7]. The microwave device was constructed using an Ampreon M2A-R semiconductor generator that emits either continuous or pulsed microwaves (maximal power, $1200 \mathrm{~W}$; microwave frequency, $2.45 \mathrm{GHz}$ ), together with an isolator (air cooling device), a power monitor, a three-stub tuner, and a short-circuit plunger. The liquid medium was continuously irradiated with microwaves through the tungsten electrode antenna (diameter, $1.0 \mathrm{~cm}$; length, $20.0 \mathrm{~cm}$ ). Metal cones were used in the waveguide to focus the microwaves onto the tip of the antenna electrode, through which the microwaves irradiated the aqueous solution and generated the in-liquid plasma (Fig. 1b). The tungsten antenna was isolated from both the reactor and the waveguide using a ceramic spacer, so that the microwave irradiated only the liquid. The cylindrically shaped reactor consisted of a quartz vessel (diameter, $32.0 \mathrm{~mm}$; length, $160.0 \mathrm{~mm}$ ) and fabricated such that the pressure reduced with a water aspirator through the top of the reactor. A three-stub tuner adjusted the irradiating microwaves such that there were no reflected waves.

\subsection{Application in wastewater treatment}

High-purity grade tetrabromobisphenol-A (TBBPA) was purchased from Wako Pure Chemical Industries, Ltd. The aqueous TBBPA solution $(50 \mathrm{~mL} ; 0.10 \mathrm{mM} \approx 54.4 \mathrm{mg}$ $\mathrm{L}^{-1}$ ) was placed in the quartz reactor and subsequently subjected to irradiation with the nerated in-liquid plasma [8]. The solution was adjusted to $\mathrm{pH}=9,11$ and 13 with sodium hydroxide. Samples of the decomposed TBBPA solution were collected at various time intervals and then analyzed to assess the extent of debromination of the TBBPA (formation of Br- ions) using a Thermo Scientific ${ }^{\mathrm{TM}}$ Dionex $^{\mathrm{TM}}$ Integrion $^{\mathrm{TM}}$ HPIC ${ }^{\mathrm{TM}}$; loss of total organic carbon (TOC) was determined using a Shimadzu TOC-L analyser. Computer simulations were carried out using a CAChe Worksystem version 3.2 (Fujitsu Co. Ltd) implemented on an Intel P-III and Windows 7 system. Frontier electron densities for radical attack in the heteroaromatic substrate was also calculated by a MOPAC/PM3 wavefunction. A geometrical configuration was determined by pre-optimization calculation in the Mechanics using augmented MM3, followed by geometrically optimized calculation in MOPAC using PM3 parameters; solvation effects in water were also simulated using COSMO. 
Intermediates species formed during the debromination and mineralization of TBBPA were identified by direct injection into the mass spectral detector of a JEOL Ltd., JMS-T100LC LC-TOFMS (electrospray ionization: ESI) system operated in the negative ion mode; orifice voltage was $30 \mathrm{~V}$. The eluent was a solution of methanol. Note that the LC column was not used in this study. The spectrometer was scanned from $\mathrm{m} / \mathrm{z}=100$ to 10,000 for recording mass spectra.

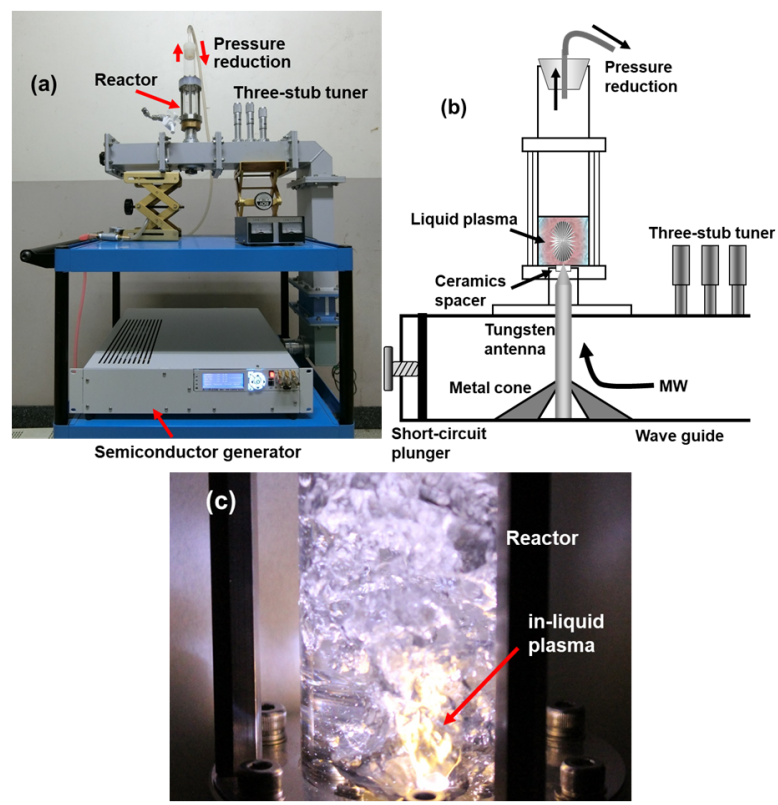

Fig. 1. (a) Photograph and (b) schematic image of in-liquid plasma device, and (c) photograph of the generated in-liquid plasma in the reactor

\subsection{Application in crosslinking agent/initiator-free polymer-gel synthesis}

High-purity grades of dichlorodimethylsilane and polyvinylpyrrolidone K30 (PVP) as starting materials for silicone gel (HySi-gel) and polyvinylpyrrolidone gel (PVP-gel) were purchased from Tokyo chemical industry Co., Ltd. In the synthesis of HySi-gel, dichlorodimethylsilane was used for monomer of dimethylpolysiloxane (silicone). $10 \mathrm{~g}$ dichlorodimethysilane was added in $50 \mathrm{~mL}$ ionized water and was stirred at room temperature $\left(25^{\circ} \mathrm{C}\right)$ for $1 \mathrm{~h}$. After that, the solution was stirred in $60^{\circ} \mathrm{C}$ for $5 \mathrm{~h}$ and silicone was synthesized in ionized water. Silicone-gel was synthesized from the silicone solution by using in-liquid plasma method at $30 \mathrm{sec}$-irradiation and $150 \mathrm{~W}$ of microwave power in the same pulse-microwave condition of the wastewater treatment. In the synthesis of PVP-gel, $3 \mathrm{~g}$ PVP was dissolved in $30 \mathrm{~mL}$ ionized water and PVP-gel was synthesized from the PVP solution by using in-liquid plasma method at 5 min-irradiation and $150 \mathrm{~W}$ of microwave power in the same pulse-microwave condition of wastewater treatment. the functional groups of PVP (before in-liquid plasma treatment) and PVP-gel (after in-liquid plasma treatment) were analyzed by using FT-IR. 


\section{Experimental results and discussions}

\subsection{Development of in-liquid plasma device}

The pulsed condition of microwave power was investigated to generate more stable plasma in aqueous solution and to extend the life of the electrode. The investigation parameters were amplitude of microwave power pulses, pulse width and pulse period. Maintaining the minimum threshold of the microwave applied power at $380 \mathrm{~W}$, the pulse width and the pulse period were then altered to achieve optimal conditions with which to generate in-liquid plasma. A matrix of the results to generate in-liquid plasma under the various conditions are presented in Table 1.

Table 1. Status of in-liquid plasma generation under different pulse conditions for a pulse period (ms : milisecond) and percent pulse duration (period) at afixed applied microwave power of $380 \mathrm{~W}$ (pulse amplitude) NG: no generation of in-liquid plasma, G: generation of in-liquid plasma, S: slight generation of sound waves, B: generation of fine bubbles

\begin{tabular}{|c|c|c|c|c|c|c|c|c|c|}
\hline \multirow{2}{*}{ Pulse period / ms } & \multicolumn{9}{|c|}{ Period /\% } \\
\cline { 2 - 10 } & 90 & 80 & 70 & 60 & 50 & 40 & 30 & 20 & 10 \\
\hline 10.0 & NG & NG & NG & NG & NG & NG & NG & NG & NG \\
\hline 11.0 & NG & NG & NG & NG & NG & NG & NG & NG & NG \\
\hline 12.5 & NG & NG & NG & NG & NG & NG & NG & NG & NG \\
\hline 14.3 & NG & NG & NG & NG & NG & NG & NG & NG & NG \\
\hline 16.7 & G & G & G & G & G & S & S & S & S \\
\hline 20.0 & S & S & S & S & S & S & B & B & B \\
\hline 25.0 & B & B & B & B & B & B & B & B & B \\
\hline
\end{tabular}

Only experiments conducted at a pulse width of $16.7 \mathrm{~ms}$ and a pulse period at $50 \%(8.35$ $\mathrm{ms})$ through $90 \%(15.03 \mathrm{~ms})$ led to the generation of stable plasma in the liquid. Consideration of power consumption, the optimal pulse irradiation conditions were a microwave irradiation power of $380 \mathrm{~W}$ (pulse amplitude), a pulse period of $16.7 \mathrm{~ms}$ and a pulse width of $13.4 \mathrm{~ms}$ (see Fig. 2). Stable generated in-liquid plasma could be sustained for $1 \mathrm{~h}$ with no visible deterioration of the tungsten antenna tip observed under these optimized conditions. Summary, we clarified the device conditions and the microwave irradiation conditions that can use the in-liquid plasma continuously for practical use, and this enabled stable experiments.

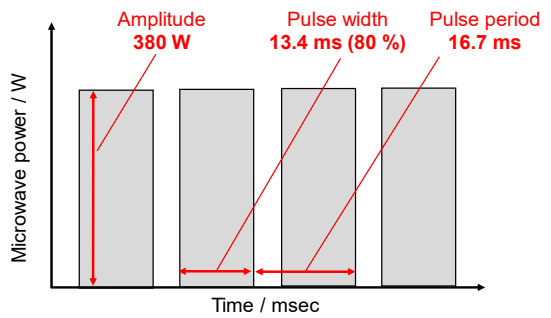

Fig. 2. Optimized conditions for pulsed microwave irradiation with a semiconductor microwave generator

\subsection{Application in wastewater treatment}

The time course of the debromination of aqueous TBBPA solutions under various alkaline conditions of $\mathrm{pH}(\mathrm{pH} \sim 9,11$ and 13) was examined with the optimised in-liquid plasma system (Fig. 1a) [8]. When decomposition was carried out at $\mathrm{pH} 13$ for an irradiation time of $20 \mathrm{~min}$, complete debromination occurred (ca. 100\%), whereas at pHs 11 and 9, the 
extent of debromination was $87 \%$ and $83 \%$, respectively (see Fig. 3a). Debromination followed first-order kinetics: $k=0.107 \pm 0.008 \mathrm{~min}^{-1}(\mathrm{pH} \mathrm{13}), 0.079 \pm 0.003 \mathrm{~min}^{-1}(\mathrm{pH} 11)$, and $0.079 \pm 0.006 \mathrm{~min}^{-1}(\mathrm{pH}$ 9). By comparison, the extent of mineralization of TBBPA was also complete $(100 \%)$ within 20 min of irradiation at $\mathrm{pH} \mathrm{13,} \mathrm{while} \mathrm{the} \mathrm{extent} \mathrm{of} \mathrm{TOC} \mathrm{loss}$ was $81 \%$ at $\mathrm{pH} 11$ and $76 \%$ at $\mathrm{pH} 9$; the corresponding first-order kinetics were: $k=0.28 \pm$ $0.05 \mathrm{~min}^{-1}$ (pH 13), $0.37 \pm 0.08 \mathrm{~min}^{-1}$ (pH 11), and $0.39 \pm 0.11 \mathrm{~min}^{-1}$ (pH 9) - see Fig. 3b. Clearly, the optimal condition to degrade and mineralize the TBBPA flame retardant is in aqueous alkaline media at $\mathrm{pH} 13$.
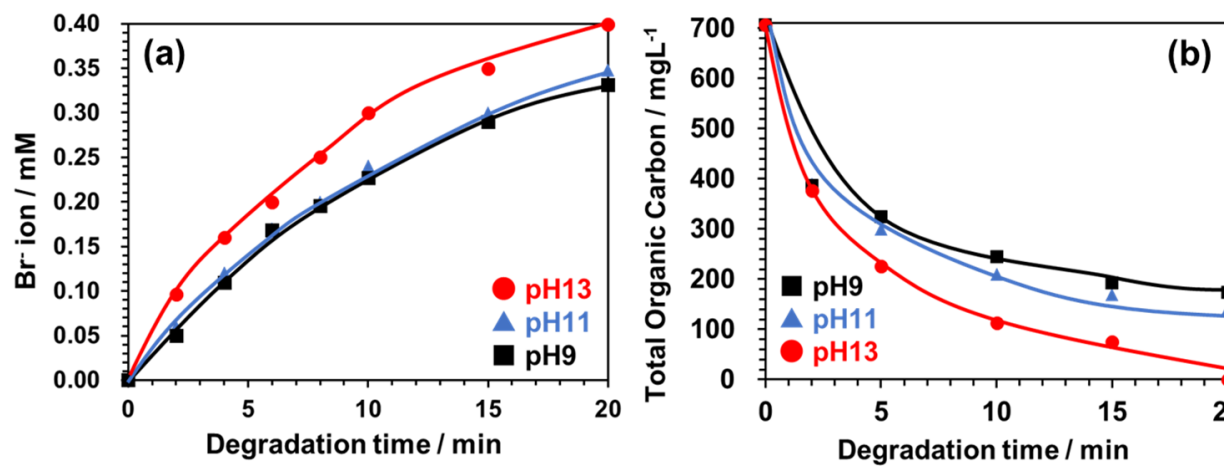

Fig. 3. (a) Dependence of the debromination of tetra-bromobisphenol-A (TBBPA) at various alkaline $\mathrm{pHs}(\mathrm{pH}=9,11$ and 13) and on irradiation time; (b) plot showing the time course of the loss of total organic carbon (TOC) under in-liquid plasma irradiation at various $\mathrm{pHs}$ of 9,11 , and 13 . Other conditions: volume of aqueous medium, $50 \mathrm{~mL}$; concentration of TBBPA, $0.10 \mathrm{mM}$ or ca. $54.4 \mathrm{mg} \mathrm{L}^{-1}$

In the graph of Fig. 3a, bromo-ion concentration of the samples depended on the $\mathrm{pH}$ value. The strongest basic solution ( $\mathrm{pH} \mathrm{13)}$ promoted to desert bromo-ion from TBBPA and the solution which in-liquid plasma had treated for $20 \mathrm{~min}$ in the $\mathrm{pH}$ value of 13-desert bromo-ion completely. On the other hands, the graph of Fig. 3b was about evaluation of TOC concentration of samples. The decrease of TOC concentration promoted to be decomposed in order of $\mathrm{pH} 13, \mathrm{pH} 11$ and $\mathrm{pH} 9$ because reactions between $\mathrm{OH}$ radical and bromo-ion occurred when in-liquid plasma generates. These reactions are shown below [10]. $\mathrm{OH}$ radical was synthesized in reaction of (eq. 1) by using in-liquid plasma method. $\mathrm{Br}^{-}$ion was ionized and $\mathrm{Br}$ radical was synthesized from TBBPA in solution by using in-liquid plasma. After that, $\mathrm{BrOH}^{-}$radical could synthesized from $\mathrm{Br}$ radical and $\mathrm{Br}^{-}$ion in reaction of (eq. 2). $\mathrm{Br} 2$ - radical, $\mathrm{Br}$ radical and $\mathrm{OH}^{-}$ion were combined into $\mathrm{BrOH}^{-}$radical in reactions of (eq.3) and (eq. 4). $\mathrm{BrOH}^{-}$radical was ionized to $\mathrm{OH}$ radical and $\mathrm{Br}^{-}$ion (eq. 5) so $\mathrm{OH}$ radical promoted to decompose TBBPA in $\mathrm{pH}$ value of 13 during generating in-liquid plasma.

$$
\begin{aligned}
& \mathrm{H}_{2} \mathrm{O}+\mathrm{e}^{*} \rightarrow \mathrm{H}^{\bullet}+{ }^{\bullet} \mathrm{OH}+\mathrm{e} \\
& \mathrm{Br}^{\bullet}+\mathrm{Br}^{-} \rightleftarrows \mathrm{Br}_{2}^{--} \\
& \mathrm{BrOH}^{\bullet-} \rightleftarrows \mathrm{Br}^{\bullet}+\mathrm{OH}^{-} \\
& \mathrm{BrOH}^{--}+\mathrm{Br}^{-} \rightleftarrows \mathrm{Br}^{\circ-}+\mathrm{OH}^{-} \\
& { }^{\circ} \mathrm{OH}+\mathrm{Br}^{-} \rightleftarrows \mathrm{BrOH}^{--}
\end{aligned}
$$

The degradation mechanism of TBBPA (Scheme 1) is investigated by computer simulation and analysis of intermediates. As an initial decomposition, ROSs such as $\mathrm{OH}$ radicals generated from in-liquid plasma, attack the position of $\mathrm{C}-\mathrm{Br}$ and debromination proceeds. Then intermediates, assigned as products (II)-(IV), were identified by LC-MS. 
The product intermediates can be mainly classified into (II) triBBPA (2,6-dibromo-4-[1-(3bromo-4-hydroxyphenyl)-1-hydroxyethyl]phenol), (III) diBBPA, (IV) monoBBPA. Finally, all bromine is ionized to form (V) BPA (bis(3-bromo-4-hydroxyphenyl)methanone). Thereafter, the ring opening of the benzene ring for BPA proceeds by further ROSs attack. Along with that, intermediates (VI) (2E,6Z)-8-hydroxy-3-methyl-5-methylidene-4-oxoocta2,6-dienal, (VII) (3Z)-5-hydroxy-2-methylidenepent-3-enoic acid, (VIII) 5hydroxypentanoic acid are formed and finally mineralized into $\mathrm{CO}_{2}$ gas and water. Oxidative degradation took place through scission, debromination, hydroxylation, or cleavage of the aromatic rings to achieve the final mineralization.
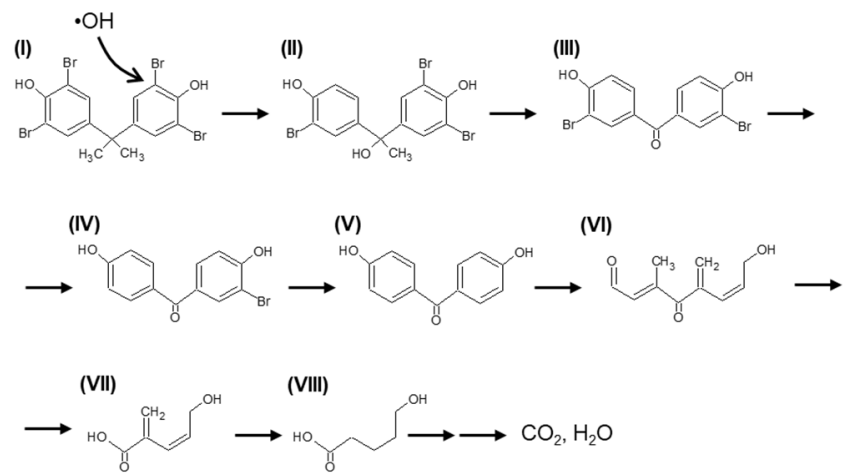

Scheme 1. Degradation pathway of tetrabromobisphenol-A with using in-liquid plasma.

Degradation of TBBPA in alkaline aqueous media by a newer advanced oxidation method - namely, the in-liquid plasma method - has been demonstrated in this study. Although there are already several oxidative decomposition methods (photocatalyst, Fenton, ROS, etc.), it has been clarified that the in-liquid plasma method can decompose them sufficiently quickly. This methodology, which is a new member of AOPs, provides a simple and green approach to detoxify TBBPA in aqueous media requiring only electric power and no such chemicals as catalysts and oxidizing agents to achieve it.

\subsection{Application in crosslinking agent/initiator-free polymer-gel synthesis}

In the case of no cross-linking agent and initiator, HySi-gel and PVP-gel is synthesized by using in-liquid plasma with $150 \mathrm{~W}$-power of microwave for $30 \mathrm{sec}$ or $5 \mathrm{~min}$ irradiation respectively.

Photographs of silicone and silicone-gel is shown in Fig. 4. Methyl groups of this gel was attacked by $\mathrm{OH}$ radical which was dissociated from $\mathrm{H}_{2} \mathrm{O}$ in reaction of (eq. 1) by inliquid plasma method and the macroradical attached with each other. Then, silicone-gel is synthesized through this process and could have some hydroxy groups because $\mathrm{OH}$ radicals attacked the silicone and combined with the gel. 


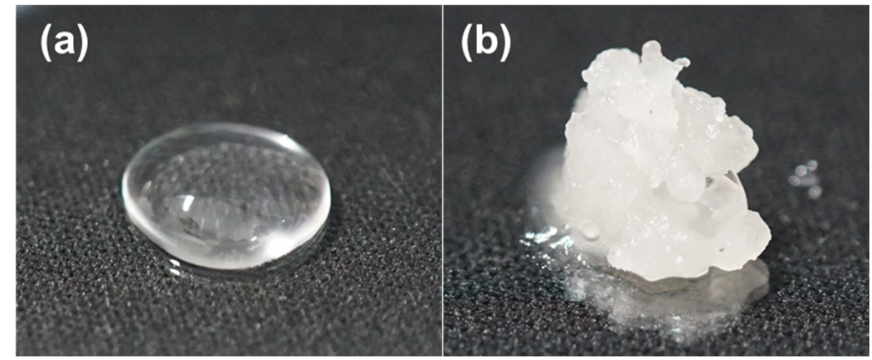

Fig. 4. Photographs of (a) silicone sample before in-liquid plasma method and (b) silicone-gel $30 \mathrm{sec}$ after in-liquid plamsa method treated silicone solution without crosslinking agent and initiator

In the condition of no cross-linking agent and initiator, PVP-gel was synthesized by using in-liquid plasma at 5 min-irradiation and $150 \mathrm{~W}$. FT-IR spectra of PVP-gel which was a sample after PVP was treated by using in-liquid plasma method and the chemical mechanism of PVP-gel synthesis are shown in Fig. 5.

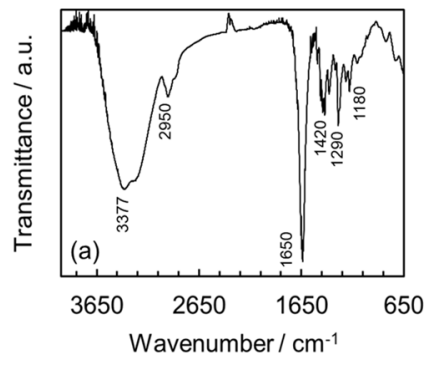

(b)
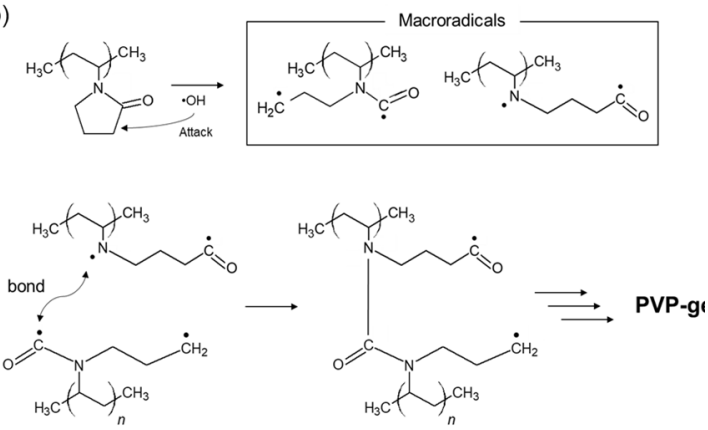

Fig. 5. Photographs of (a) FT-IR spectra of PVP-gel which was a sample 5 min after PVP was treated by using inliquid plasma method, and (b) a chemical mechanism of the PVP-gel

In the spectra (Fig. 5a), PVP-gel has hydroxy groups $\left(3377 \mathrm{~cm}^{-1}\right)$, carboxyl group $\left(2950 \mathrm{~cm}^{-}\right.$ $1,1420 \mathrm{~cm}^{-1}$ and $\left.1180 \mathrm{~cm}^{-1}\right)$ and tertiary amide group $\left(1650 \mathrm{~cm}^{-1}, 1290 \mathrm{~cm}^{-1}\right)$. According to the spectra, $\mathrm{OH}$ radical, which dissociated from $\mathrm{H} 2 \mathrm{O}$ by using in-liquid plasma, opened fivemember ring of PVP and the PVP became the macroradicals. In the chemical structure, these macroradicals attacked each other and these chemical reactions occurred for many times (Fig. 5b). After that, PVP-gel was synthesized [11].

They are the first attempt to synthesize gel only by the power of electricity. Since the initiator and crosslinking agent are very toxic materials, LP method is effective as a green chemistry process.

\section{Conclusions}

The best conditions of pulsed microwave (pulse width: $16.7 \mathrm{~ms}$, pulse period: $80 \%$ ) was investigated to generate stable plasma in liquid and was tried to use in-liquid plasma method for applications of wastewater treatment and gel-synthesis. In the application of wastewater treatment, it took only $20 \mathrm{~min}$ for in-liquid plasma method to be able to decompose and mineralize $0.1 \mathrm{M}$ TBBPA solution and in-liquid plasma method has high potential for 
wastewater treatment. In the applications of wastewater treatment, we could synthesize HySi-gel and PVP-gel without crosslinking agent and initiator. In-liquid plasma method will be a new gel-synthesis of green chemistry process.

\section{References}

1. J. S. Clements, M. Sato, R. H. Davis, IEEE Trans. Ind. Appl., 1987, 23, 224-235.

2. S. Horikoshi, N. Serpone, RSC Adv., 2017, 7, 47196-47218.

3. S. Nomura, H. Toyota, Appl. Phys. Lett., 2003, 83, 4503-4505.

4. S. Nomura, H. Toyota, S. Mukasa, Y. Takahashi, T, Maehara, A. Kawashima, H. Yamashita, Appl. Phys. Express, 2008, 1, 046002.

5. S. Horikoshi, S. Sato, M. Abe, N. Serpone, Ultrason. Sonochem., 2011, 18, 938-942.

6. A. Tsuchida, T. Shimamura, S. Sawada, S. Sato, N. Serpone, S. Horikoshi, Rad. Phys. Chem., 2018, 147, 53-58.

7. S. Horikoshi, S. Sawada, S. Sato, N. Serpone, Plasma Chem. Plasma Process., 2019, 39, 51-62

8. S. Horikoshi, S. Sawada, A. Tsuchida, N. Serpone, Environ. Sci. Water Res. Technol., 2019, submitted

9. S. Horikoshi, S. Sawada, 2019, will be submitted

10. Y. Guo, Jun Zhou, Xiaoyi Lou, Renlan Liu, Dongxue Xiao, Changling Fang, Zhaohui Wang, Jianshi Liu, Chem. Eng. J., 2014, 254, 538-544.

11. G.J.M. Fechine, J.A.G. Barrros, M.R. Alacantra, L. H. Catalani, Polymer, 2006, 47, 2629-2633. 